\title{
CURRENT SHEET REGULATION OF SOLAR NEAR-RELATIVISTIC ELECTRON INJECTION HISTORIES
}

\author{
N. Agueda ${ }^{1}$, R. VAinio ${ }^{2}$, S. Dalla $^{3}$, D. Lario ${ }^{4}$, And B. Sanahuja ${ }^{1}$ \\ ${ }^{1}$ Departament d'Astronomia i Meteorologia, Institut de Ciències del Cosmos, Universitat de Barcelona, Spain \\ ${ }^{2}$ Department of Physics, University of Helsinki, Finland \\ 3 Jeremiah Horrocks Institute, University of Central Lancashire, UK \\ ${ }^{4}$ Applied Physics Laboratory, The Johns Hopkins University, USA \\ Received 2012 May 9; accepted 2013 January 16; published 2013 February 20
}

\begin{abstract}
We present a sample of three large near-relativistic ( $>50 \mathrm{keV}$ ) electron events observed in 2001 by both the $A C E$ and the Ulysses spacecraft, when Ulysses was at high-northern latitudes $\left(>60^{\circ}\right)$ and close to 2 AU. Despite the large latitudinal distance between the two spacecraft, electrons injected near the Sun reached both heliospheric locations. All three events were associated with large solar flares, strong decametric type II radio bursts and accompanied by wide $\left(>212^{\circ}\right)$ and fast $\left(>1400 \mathrm{~km} \mathrm{~s}^{-1}\right)$ coronal mass ejections (CMEs). We use advanced interplanetary transport simulations and make use of the directional intensities observed in situ by the spacecraft to infer the electron injection profile close to the Sun and the interplanetary transport conditions at both low and high latitudes. For the three selected events, we find similar interplanetary transport conditions at different heliolatitudes for a given event, with values of the mean free path ranging from $0.04 \mathrm{AU}$ to $0.27 \mathrm{AU}$. We find differences in the injection profiles inferred for each spacecraft. We investigate the role that sector boundaries of the heliospheric current sheet (HCS) have on determining the characteristics of the electron injection profiles. Extended injection profiles, associated with coronal shocks, are found if the magnetic footpoints of the spacecraft lay in the same magnetic sector as the associated flare, while intermittent sparse injection episodes appear when the spacecraft footpoints are in the opposite sector or a wrap in the HCS bounded the CME structure.
\end{abstract}

Key words: interplanetary medium - Sun: coronal mass ejections (CMEs) - Sun: flares - Sun: particle emission

Online-only material: color figures

\section{INTRODUCTION}

In situ observations of near-relativistic (NR; $>50 \mathrm{keV})$ electron events from a single point in the heliosphere can be used to infer the mechanisms of electron acceleration at the Sun (see Kahler 2007, for a review). Multi-spacecraft observations of NR electron events from well-separated heliographic locations provide us with additional information on the processes that inject NR electrons at different heliolongitudes and heliolatitudes as well as the propagation conditions of solar energetic particles (SEPs) throughout the heliosphere.

In this paper, we analyze three large NR electron events observed by both the Advanced Composition Explorer (ACE) and the Ulysses spacecraft during its northern high-latitude passage. All three events were associated with large solar flares and accompanied by wide $\left(>212^{\circ}\right)$ and fast $\left(>1400 \mathrm{~km} \mathrm{~s}^{-1}\right)$ coronal mass ejections (CMEs). Strong type II radio bursts, often considered as a clear signature of a propagating shock (e.g., Cane et al. 1981), were reported during the three events. The main difference between the three events was the relative position between the flare site and the footpoints of the nominal magnetic field lines connecting each spacecraft to the Sun with respect to the heliospheric current sheet (HCS).

The important role of the HCS on interplanetary shock propagation was investigated by Zhao et al. (2007) in a statistical study of shock occurrence at 1 AU. They found that for a group of solar sources with similar angular distances to the spacecraft footpoint, the associated interplanetary shocks had a larger probability to reach Earth when the flare site and the nearEarth spacecraft footpoint were on the same side of the HCS, suggesting an impeding effect of the HCS on shock propagation.

MHD simulations (Steinolfson \& Mullan 1980) showed that the crossing of a neutral line can reduce the shock speed by a factor of more than two, supporting the idea that the HCS can act as a "barrier" for shock propagation. The attenuation is stronger when the neutral sheet is wider, the Mach number of the shock larger, and the magnetic field of the current sheet higher. Observational evidence of shock attenuation in a neutral sheet was provided by Mullan (1981).

Two previous observational studies have investigated the role of the HCS on shock-associated SEP acceleration (Kallenrode 1993; Kahler et al. 1996), focusing on the statistical trends of the event onset time, event peak intensity, and rise time, relative to the current sheet positions. Kallenrode (1993) provided the first statistical comparison of SEP flux profiles using multispacecraft observations of $\sim 0.5 \mathrm{MeV}$ electrons and $\sim 7 \mathrm{MeV}$ protons by Helios 1 and 2 . In their study, they concluded that the timescales of SEP events were ordered by the angular distance from the flare site to the spacecraft footpoint as well as the occurrence of sector boundaries. Kallenrode (1993) suggested a scenario in which the coronal shock front injecting particles would reach a rapid magnetic connection with two spacecraft if both were connected to the flare sector. On the other hand, magnetic connection could be achieved slowly (even at smaller angular distances) if there was a magnetic sector boundary between the spacecraft footpoint and the flare site.

In a later study, Kahler et al. (1996) looked for an indication of the HCS modulation on $>10 \mathrm{MeV}$ SEP events observed by the Interplanetary Monitoring Platform spacecraft and found no substantial differences for SEP events with solar sources on the same side of the current sheet and SEP events with sources on the opposite side relative to the observer. Kahler et al. (1996) expected later onset and peak times, as well as lower peak fluxes, when the source and the footpoint of the observer were located in different magnetic sectors rather than in same sector.

The contradictory results reported by Kallenrode (1993) and Kahler et al. (1996) could be related to the fact that both studies were based on SEP event parameters (onset time, peak time, 
Table 1

Characteristics of the SEP Events

\begin{tabular}{|c|c|c|c|c|c|c|c|c|c|c|c|}
\hline \multirow[b]{2}{*}{ Date } & \multirow[b]{2}{*}{$\mathrm{S} / \mathrm{C}$} & \multirow{2}{*}{$\begin{array}{c}\text { Radial } \\
\text { Distance } \\
(\mathrm{AU})\end{array}$} & \multicolumn{2}{|c|}{ HGI } & \multirow{2}{*}{$\begin{array}{c}\text { Solar Wind } \\
\qquad u\rangle \\
\left(\mathrm{km} \mathrm{s}^{-1}\right)\end{array}$} & \multicolumn{5}{|c|}{ Electron Event } & \multirow{2}{*}{$\begin{array}{c}\text { Shock } \\
\text { Passage } \\
\text { (UT) }\end{array}$} \\
\hline & & & $\begin{array}{c}\text { Latitude } \\
\left(^{\circ}\right)\end{array}$ & $\begin{array}{c}\text { Longitude } \\
\left({ }^{\circ}\right)\end{array}$ & & $\begin{array}{c}\text { Onset E'4 } \\
\text { (UT) }\end{array}$ & $\begin{array}{c}\text { Period } \\
\text { (UT) }\end{array}$ & $n$ & $S$ & $\begin{array}{c}\langle\mu-\operatorname{co}\rangle \\
(\%)\end{array}$ & \\
\hline \multirow[t]{2}{*}{$2001 / 267$} & A & 1.0 & 7 & 285 & 450 & $10: 52$ & {$[10: 42,14: 42]$} & 202 & 3.5 & 85 & 268/20:50 \\
\hline & $\mathrm{U}$ & 1.9 & 78 & 317 & 700 & $15: 15$ & {$[15: 00,23: 00]$} & 122 & 1.1 & 92 & $270 / 21: 22$ \\
\hline \multirow[t]{2}{*}{$2001 / 308$} & A & 1.0 & 4 & 326 & 350 & $16: 36$ & {$[16: 26,20: 26]$} & 202 & 2.8 & 94 & 309/01:55 \\
\hline & $\mathrm{U}$ & 2.2 & 77 & 30 & 700 & $18: 43$ & {$[18: 30,01: 00]$} & 100 & 1.0 & 78 & 312/06:53 \\
\hline \multirow[t]{2}{*}{$2001 / 360$} & A & 1.0 & -2 & 18 & 400 & $05: 25$ & {$[05: 30,09: 00]$} & 177 & 3.0 & 78 & $363 / 04: 47$ \\
\hline & $\mathrm{U}$ & 2.5 & 67 & 57 & 600 & 07:11 & {$[06: 30,14: 00]$} & 110 & 1.4 & 79 & $\cdots$ \\
\hline
\end{tabular}

and rise time) that are determined by the properties of the interplanetary transport as well as by the onset and time of maximum of the injection profile.

In this study, we utilize advanced interplanetary transport modeling and make use of the directional intensities observed in situ by the $A C E$ and Ulysses spacecraft to infer the electron injection profile close to the Sun (Agueda et al. 2008, 2009a, 2012a). For each event, we study the relative positions between the flare source and the nominal footpoints of the two spacecraft with respect to the location of the HCS. We investigate the effects of the HCS on SEP release by comparing the inferred injection profiles.

In Section 2, we describe the in situ particle observations and the parent solar activity associated with each SEP event. In Section 3, we review the transport model and the inversion method that we use to fit the spacecraft observations. We present the main results in Section 4 and discuss the main implications in Section 5. The summary is presented in Section 6.

\section{OBSERVATIONS AND EVENT SELECTION}

\subsection{Near-relativistic Electron Events at ACE and Ulysses}

We use NR electron measurements by the Heliosphere Instrument for Spectra Composition and Anisotropy at Low Energies (HI-SCALE) on board Ulysses (Lanzerotti et al. 1992) and compare them with in-ecliptic measurements by the Electron Proton and Alpha Monitor (EPAM) on board the $A C E$ spacecraft (Gold et al. 1998). Since ACE/EPAM was the spare instrument of Ulysses/HI-SCALE, particle data from both detectors are directly comparable.

We present results for NR electrons in the E'4 energy channel of both experiments (175-312 keV for ACE/EPAM and 178-290 keV for Ulysses/HI-SCALE), with a mean energy of $230 \mathrm{keV}$; such electrons have a mean speed of $0.7 c$ and take $\sim 11$ minutes to travel $1 \mathrm{AU}$ under scatter-free conditions. We make use of the intensities measured by the LEFS60 telescope on board Ulysses and ACE. Both spacecraft are spin-stabilized with a spin period of $12 \mathrm{~s}$ and the spacecraft rotation is used to scan with a single detector different directions of space. The swath of space swept out by the LEFS60 telescope during a spin is divided into eight equally spaced sectors. In this study, the data were obtained with $72 \mathrm{~s}$ time resolution for $A C E$ and 4 minute time resolution for Ulysses, averaging over 6 and 20 spin periods, respectively.

We selected three NR electron events observed by both ACE and Ulysses on 2001 September 24 (DOY 267), 2001 November 4 (DOY 308), and 2001 December 26 (DOY 360). These three events show for both spacecraft (1) evidence of velocity dispersion during the rising phase of the event, (2) at least one order of magnitude between the pre-event background intensities and the event peak intensity, and (3) good coverage in pitch-angle, which guarantees that the observations contain enough directional information for their simulation (Agueda et al. 2009b). The three events under study are summarized in Table 1 which gives the date of each electron event (Column 1); the spacecraft (Column 2, A for ACE, U for Ulysses); the spacecraft location (Columns 3-5, radial distance, latitude, and longitude in the heliographic inertial (HGI) coordinate system); the mean measured solar wind speed at the onset of the event (Column 6); the electron event characteristics (Columns 7-11, onset time, fitting period, number of selected time points for fitting (see Section 3), strength, and mean pitch-angle coverage); and the time of shock passage ${ }^{5,6}$ (Column 12). The strength of the event, $S$, (Column 10) is defined as the logarithm of the ratio between the spin-averaged peak intensity and the preevent background. The pitch-angle coverage, $\mu$-co, (Column 11) is defined as the percentage of the pitch-angle cosine range scanned by the telescope (Agueda et al. 2009b).

The selected events were observed by Ulysses when it was at high-northern heliolatitudes $\left(\Lambda>65^{\circ}\right)$, at radial heliocentric distances between 1.9 and $2.5 \mathrm{AU}$, and embedded in a fast solar wind flow (with a mean speed $\geqslant 600 \mathrm{~km} \mathrm{~s}^{-1}$ ) from the northern polar coronal hole where only an inward magnetic field polarity was observed (McComas et al. 2002). On the other hand, $A C E$ was in orbit around the L1 Sun-Earth libration point and embedded in a slow solar wind stream (with a mean speed $\leqslant 450 \mathrm{~km} \mathrm{~s}^{-1}$ ).

For each event, we selected a period of at least $4 \mathrm{hr}$ after the onset, during which the source of NR electrons could be assumed to be close to the Sun. The pitch-angle cosine coverage of the observations is on average $\geqslant 70 \%$. An in situ shock passage was registered in all cases but for the 2001/360 event at Ulysses.

Figure 1 shows the spin-averaged intensities observed by $A C E$ and Ulysses for the selected electron events. The four lower panels show the solar wind speed, the direction $(\theta$ is the latitude and $\phi$ is the longitude in the Radial Tangential Normal (RTN) coordinate system centered at each spacecraft), and magnitude of the interplanetary magnetic field. The onset and time of maximum of the events were observed several hours later at Ulysses than at $A C E$ and the maximum intensity was decreased at Ulysses (Lario et al. 2003). The onset time delays at Ulysses are much longer than those expected for $\sim 230 \mathrm{keV}$ electrons traveling scatter-free up to $2.5 \mathrm{AU}$. The pitch-angle distributions (PADs) at the onset of these events showed stronger antisunward anisotropic flows at ACE than at Ulysses (Lario et al. 2003).

\footnotetext{
5 http://www-ssg.sr.unh.edu/mag/ace/ACElists/obs_list.html

6 http://www.sp.ph.ic.ac.uk/Ulysses/shocklist.txt
} 

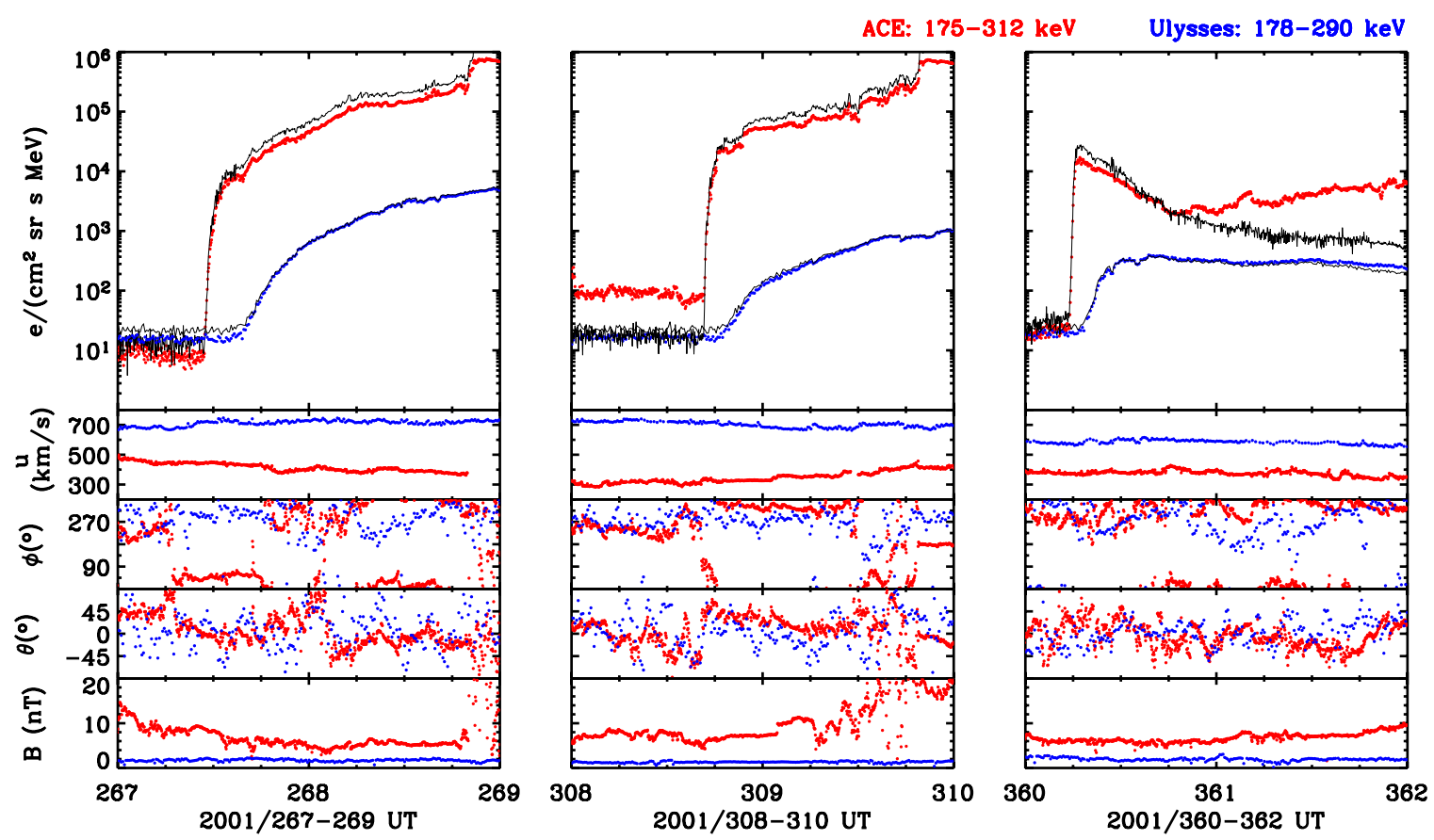

Figure 1. Electron events. For each event (from top to bottom): electron spin-averaged intensities observed by the LEFS60 telescope of ACE (red) and Ulysses (blue); magnetically deflected electron intensities from the LEMS30 telescope in approximately the same energy ranges (thin). Solar wind speed observed by each spacecraft. Magnetic field longitude $(\phi)$, latitude $(\theta)$, and magnitude measured in the RTN coordinate system.

(A color version of this figure is available in the online journal.)

Table 2

Electromagnetic Emissions Associated with the NR Electron Events

\begin{tabular}{|c|c|c|c|c|c|c|c|c|}
\hline \multirow[b]{2}{*}{ Date } & \multicolumn{3}{|c|}{ Soft X-Rays } & \multirow[b]{2}{*}{$\begin{array}{c}\mathrm{H} \alpha \\
\text { Position }\end{array}$} & \multirow{2}{*}{$\begin{array}{l}\text { Radio } \\
\text { Type II } \\
\text { (UT) }\end{array}$} & \multicolumn{3}{|c|}{ White-light CME } \\
\hline & $\begin{array}{l}\text { Start } \\
\text { (UT) }\end{array}$ & $\begin{array}{l}\text { Rise } \\
(\mathrm{min})\end{array}$ & Class & & & $\begin{array}{c}\text { First Obs. } \\
\text { (UT) }\end{array}$ & $\begin{array}{c}\text { Speed } \\
\left(\mathrm{km} \mathrm{s}^{-1}\right)\end{array}$ & $\begin{array}{l}\text { Width } \\
\left({ }^{\circ}\right)\end{array}$ \\
\hline $2001 / 267$ & $09: 32$ & 97 & $\mathrm{X} 2.6$ & S16E23 & $267 / 10: 45-268 / 20: 00$ & $10: 31$ & 2402 & Halo \\
\hline $2001 / 308$ & $16: 03$ & 54 & $\mathrm{X} 1.8$ & N06W18 & $308 / 16: 30-310 / 11: 00$ & $16: 35$ & 1810 & Halo \\
\hline $2001 / 360$ & $04: 32$ & 68 & M7.1 & N08W54 & $360 / 05: 20-361 / 05: 00$ & $05: 30$ & 1446 & $>212$ \\
\hline
\end{tabular}

\subsection{Solar Origin}

Table 2 lists the solar origin associated with each event. It gives the date of the flare (Column 1); the start and rise time of the soft X-ray emission (Columns 2-3); the flare class (Column 4); the $\mathrm{H} \alpha$ flare position (Column 5); the timing of the type II radio bursts (Column 6) observed by Wind/WAVES ${ }^{7}$ (Bougeret et al. 1995); and the CME parameters (Columns 7-9), as reported in the $\mathrm{SOHO} / \mathrm{LASCO} \mathrm{CME}$ catalog $^{8}$ (time of the first appearance in the $\mathrm{C} 2$ coronagraph, the plane-of-sky speed of the leading edge and the angular width).

The three events were associated with gradual intense solar flares with rise times larger than $\sim 1 \mathrm{hr}$ and GOES X-classes larger than M7. The CMEs observed in association were all fast (plane-of-sky speeds larger than $1400 \mathrm{~km} \mathrm{~s}^{-1}$ ) and wide (two halos, and one $\mathrm{CME}$ wider than $212^{\circ}$ ). Intense decametric type II radio bursts $(14-0.03 \mathrm{MHz}$ ) were reported for the three events (Gopalswamy et al. 2002; Cane \& Erickson 2005).

Figure 2 shows the source surface magnetic fields at $2.5 R_{\odot}$ provided by The Wilcox Solar Observatory. ${ }^{9}$ The coronal magnetic field is calculated from photospheric field observations with a potential field model (PFSS; Schatten et al. 1969;

\footnotetext{
7 http://www-lep.gsfc.nasa.gov/waves/bursts_2001.html

8 http://cdaw.gsfc.nasa.gov/CME_list/

9 http://wso.stanford.edu/
}

Hoeksema et al. 1983), assuming that the field is radial at $2.5 R_{\odot}$, and in good agreement with the polarity pattern observed at Earth. These maps only show the large-scale field, the sectors observed in interplanetary space, while the small-scale complex magnetic field structures of the active regions are averaged out. The thick black curve identifies the heliospheric current sheet separating both polarities at $2.5 R_{\odot}$. Figure 2 shows for each event the flare location at the solar surface (black cross) and the spacecraft footpoints at the source surface (red cross for $A C E$ and blue cross for Ulysses), calculated using the solar wind speed measured in situ at the onset of each event and assuming an Archimedean interplanetary magnetic field (IMF).

For all the events, the footpoints of the two spacecraft lie in the northern hemisphere of the Sun, suggesting that the root of the IMF field lines at the source surface had an inward polarity. On the other hand, the three events show very different spatial configurations on the source surface map. For the 2001/308 event (middle panel), the flare and the footpoints of both spacecraft lie within the same magnetic field sector. For the $2001 / 267$ event (top panel), both spacecraft are connected to a sector different from the sector where the flare occurred. Finally, for the 2001/360 event (bottom panel), the flare and the footpoints of both spacecraft lie within the same magnetic field sector but a wrap in the HCS indicates the presence of a neutral line between the flare site and the Ulysses footpoint. 

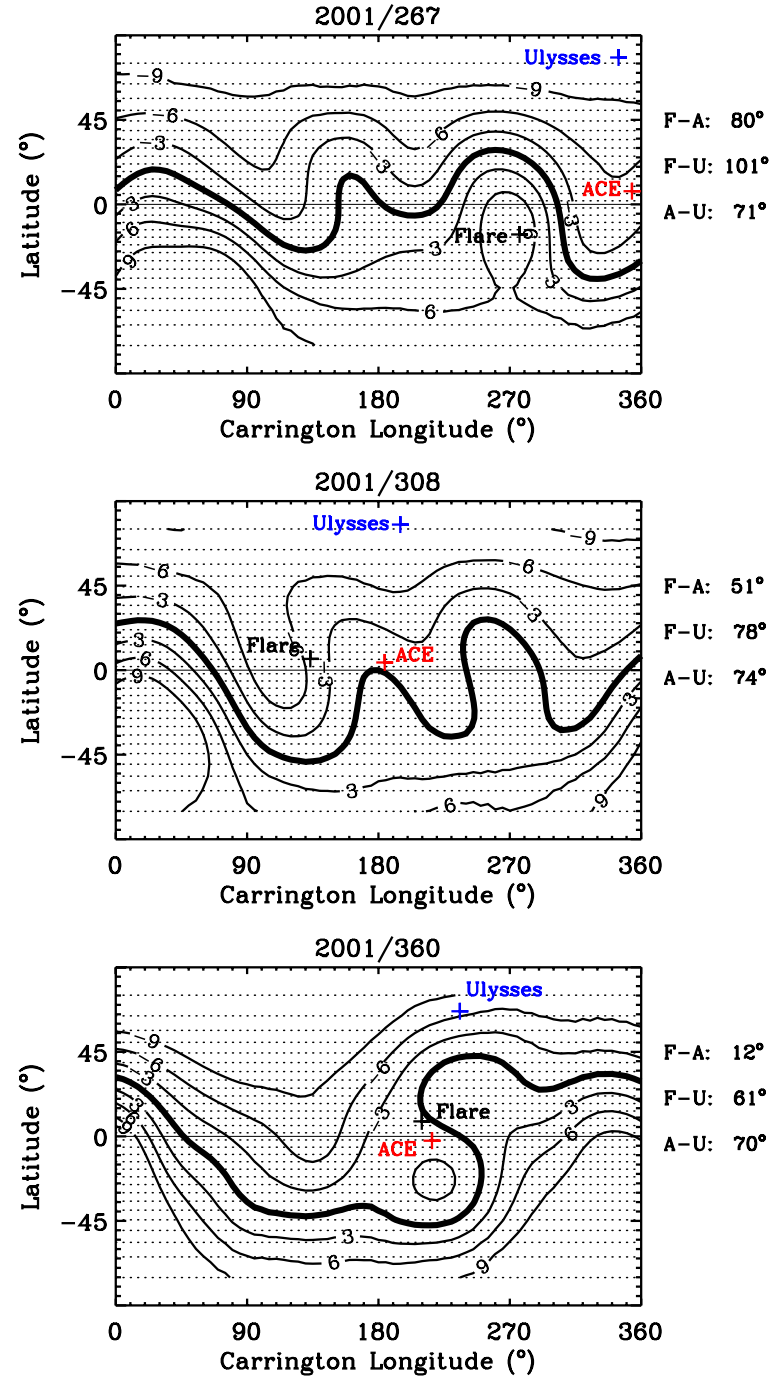

Figure 2. Source surface magnetic field at $2.5 R_{\odot}$, heliospheric current sheet (thick black curve), position of the flare at the solar surface (black cross), and spacecraft footpoints (blue and red crosses) for ACE and Ulysses, respectively. For each event, the angular distances on the sphere between the flare $(F)$, the $A C E$ (A) and the Ulysses (U) footpoints are listed.

(A color version of this figure is available in the online journal.)

For each event, we calculated the angular distance over the sphere from the flare site to the two spacecraft footpoints, in addition to the angular distance from the $A C E$ footpoint to the Ulysses footpoint (see Figure 2). For the three events, the angular distance between the two spacecraft footpoints is about $70^{\circ}$. The angle from the flare site to the $A C E$ footpoint ranges from $12^{\circ}$ to $80^{\circ}$. The angle from the flare site to the Ulysses footpoint ranges from $61^{\circ}$ to $101^{\circ}$.

\section{MODELING OF TRANSPORT AND INJECTION}

We used the energetic particle transport model of Agueda et al. (2008) to simulate the propagation of NR electrons along the IMF. This model allows us to compute the PADs of electrons at a given spacecraft location resulting from an instantaneous solar injection close to the Sun, i.e., the Green's functions of interplanetary transport. The particle propagation model is based on the focused transport equation that includes the effects of particle streaming along the magnetic field lines, the effects of adiabatic focusing by the diverging IMF (Roelof 1969), the interplanetary scattering by magnetic fluctuations frozen into the solar wind (Jokipii 1966; Dröge 2003), the convection with scattering fluctuations, and the adiabatic deceleration resulting from the interplay of scattering and focusing (Ruffolo 1995). In our simulations, transport perpendicular to the large-scale magnetic field (see, e.g., Dröge et al. 2010; Kelly et al. 2012) was neglected.

As initial condition, electrons are released instantaneously at a distance of two solar radii from the center of the Sun following a power law in energy. The model assumes an Archimedeanspiral magnetic flux tube connecting the Sun and the spacecraft, consistent with the solar wind speed observed in situ.

In this work, we assume isotropic pitch-angle scattering and use the radial mean free path, $\lambda_{r}$, as the only free parameter to describe the pitch-angle scattering processes undergone by the energetic particles (more details can be found in Agueda et al. 2008). Based on previous works (e.g., Kallenrode et al. 1992), we assume that it is a good approximation to take $\lambda_{r}$ to be spatially independent.

The estimation of the injection function is an inversion problem in which data measured at the spacecraft position are the known "response" of the transport process, which at the same time is a convolution of the Green's function of interplanetary transport and the solar injection function. Thus, the inversion problem is to determine the best-fit injection function subject to the constraint that it must be a non-negative function (see Agueda et al. 2008, for more details). We use the non-negative least-squares (NNLS) method developed by Lawson \& Hanson (1974) to infer the injection profile for a given value of $\lambda_{r}$. Uncertainties in the data are not taken into account in the fit. These are a combination of statistical (generally negligible) and systematic errors which are not easy to estimate.

The duration of the injection function $t \in\left[t_{1}^{\prime}, t_{2}^{\prime}\right]$ is determined by the SEP event time interval selected for the study $t \in\left[t_{1}, t_{2}\right]$, that is, $t_{1}^{\prime}=t_{1}-\Delta t$ and $t_{2}^{\prime}=t_{2}-\Delta t$, where $\Delta t$ is the electrons' transit time from the Sun to the spacecraft for a given value of $\lambda_{r}$. The number of time points in the event time interval selected for fitting is equal to $n=\left(t_{2}-t_{1}\right) / \delta t+2$, where $\delta t$ is the time resolution of the data. Thus, we compute $n$ sectored Green's functions corresponding to $n$ different delta injection times. The total number of observational points is $8 n$ (because the telescopes have eight sectors) and the total number of independent fitting parameters is $n$. Therefore, the NNLS problem is well determined. The results of the inversion problem correspond to a comb of $n$ discrete delta-function injection amplitudes at different times (shown in histogram form in Figure 4). The time resolution of the injection profile is given by the time resolution of the data used for fitting.

In order to be able to invert spacecraft observations, with sometimes an incomplete scanning of the PADs, it is necessary to use the angular response of each sector to transform the simulated Green's functions into sectored Green's functions (Agueda et al. 2008, 2009b). Finally, to determine the best-fit set of $\lambda_{r}$ and injection function, we minimize a goodness-offit estimator that computes the sum of logarithmic differences between the observational and the modeled data (Agueda et al. 2008).

It is important to point out that since the model assumes a static source at two solar radii, the effects of a moving source are not taken into account. However, the incurred error is small as long as the travel time of NR electrons from two solar radii to the actual source position is small compared to the electron propagation time to the spacecraft, and smaller than the time 
Table 3

Inversion Results for Three Events Observed by Both ACE and Ulysses

\begin{tabular}{lcccc}
\hline \hline \multirow{2}{*}{ Date } & & & \multicolumn{2}{c}{ Injection } \\
\cline { 4 - 5 } & S/C & $\lambda_{r}$ & $\begin{array}{c}\text { Onset } \\
(\mathrm{UT})\end{array}$ & Comment \\
\hline $2001 / 267$ & $\mathrm{~A}$ & 0.04 & $10: 33$ & Sparse \\
$2001 / 308$ & $\mathrm{U}$ & 0.08 & $14: 40$ & Sparse \\
& $\mathrm{A}$ & 0.24 & $16: 27$ & Extended \\
$2001 / 360$ & $\mathrm{U}$ & 0.27 & $18: 08$ & Extended \\
& $\mathrm{A}$ & 0.19 & $05: 25$ & Extended \\
& $\mathrm{U}$ & 0.19 & $05: 54$ & Sparse \\
\hline
\end{tabular}

resolution of the particle data used for the study (72 s for $A C E$ and 4 minutes for Ulysses data). As long as the injecting source is below $22 R_{\odot}\left(65 R_{\odot}\right)$, the incurred delay is smaller than the $A C E$ (Ulysses) time resolution of the data used in this study.

\section{RESULTS}

Table 3 lists the best-fit values of $\lambda_{r}$ obtained by assuming that the particle mean free path is uniform along the magnetic flux tube. For the selected events, the values of $\lambda_{r}$ range from 0.04 to $0.27 \mathrm{AU}$. The values of $\lambda_{r}$ obtained for different spacecraft are similar for the same event, which suggests similar interplanetary transport conditions for the NR electrons observed at high and low heliolatitudes. Figure 3 shows the best-fit time-intensity profiles for the selected events. For simplicity, only data from three different sectors are displayed; those scanning particles mainly antisunward (top panel), perpendicular (middle panel), and sunward (bottom panel) to the mean magnetic field (note that the IMF polarity was assumed to be -1 ).

The fits succeed in reproducing most of the features of the directional intensities observed by both spacecraft. Only for two events, the 2001/267 and the 2001/308 events observed by $A C E$, do small discrepancies appear between the modeled intensities and the observations. For the event observed on $2001 / 267$ by $A C E$, there is a timing discrepancy at the onset in the sector scanning particles mostly with $\mu \simeq 0$. Modeled intensities predict an earlier arrival of particles with these pitchangle cosines, while observations show a later onset. This difference suggests that the interplanetary scattering processes might have been more selective with $\mu \simeq 0$. By assuming an anisotropic pitch-angle diffusion coefficient (instead of an isotropic one) the results of the event inversion explain better, but not completely, the intensities observed in sector 6; the modeled sectored intensities start to rise 7 minutes before the observed onset. The best fit is obtained for $\lambda_{r}=0.04 \mathrm{AU}$ and the injection function (not shown here) is sparse and very similar to the one inferred for the isotropic case. We believe that the largest source of discrepancy for this event is the non-Archimedean local magnetic field configuration (at least $90^{\circ}$ off the nominal direction for most of the event) that does not seem to be consistent with the assumptions made in the model. For the event observed on $2001 / 308$ by $A C E$, the local magnetic field direction is consistent with that of the model until 18:15 UT, when the field showed a northward excursion.

Figure 4 shows for each event and from top to bottom (a) the soft X-ray (SXR; 1-8 $\AA$ ) flux and the time-height profile of the associated $\mathrm{CME}$, (b) the electron injection profile inferred for $A C E$, (c) the electron injection profile inferred for Ulysses, and (d) the radio flux observed by Wind/WAVES, together with the frequency of emission of the plasma at the height of the CME leading edge, if the density model of Vršnak et al. (2004) is used. The duration of the inferred injection profiles is limited by gray rectangles. For comparison purposes, electron injection times have been shifted by $500 \mathrm{~s}$ to account for the travel time of solar electromagnetic emission to 1 AU (from now on, all injection times reported in the text also include this shift).

For the 2001/267 event, the electron injection begins at 10:33 UT in the flux tube connecting $A C E$ to the Sun, 36 minutes before the peak of the SXR emission and 2 minutes after the time of the CME first appearance in the $\mathrm{C} 2$ coronagraph, when the CME was at $3 R_{\odot}$. The electron injection at high latitudes was delayed. It started at 14:40 UT in the flux tube connecting Ulysses to the Sun, $\sim 4 \mathrm{hr}$ after the beginning of the injection for $A C E$. The inferred injection profile is very sparse for both spacecraft, in the sense that there are long gaps between subsequent injection episodes. We define a gap as a time interval with consecutive zero injection amplitudes. For these injection profiles, the average gap period between injection episodes is 19 minutes for $A C E$ and 32 minutes for Ulysses, and the gap distribution is broad, with gaps up to 50 minutes. During the selected time periods, the maximum injection rate was eight times larger for $A C E$ than for Ulysses.

For the 2001/308 event, the electron injection in the flux tube connecting $A C E$ to the Sun begins at 16:27 UT and extends for more than $2 \mathrm{hr}$. The injection onset occurred 30 minutes before the peak in SXR emission and 3 minutes before the beginning of the reported type II radio emission. Eight minutes later the $\mathrm{CME}$ was first observed in the $\mathrm{C} 2$ coronagraph at $4 R_{\odot}$. Electron injection in the flux tube connecting Ulysses to the Sun started at 18:08 UT, $\sim 1.5 \mathrm{hr}$ later than for $A C E$, and it extended for several hours $(>3 \mathrm{hr})$. The injection profiles for both spacecraft extend for several hours, with a mean gap period between injection amplitudes of 3 minutes for $A C E$ and of 11 minutes for Ulysses. The two gap distributions extend up to 26 minutes and they both peak at the mean gap value. If we convolve the modeled Green functions with the smoothed injection profiles (thick red/blue curves in Figure 4), the obtained fit does not practically differ from the best fit, which suggests that the inferred short injection gaps should not be regarded as physical and could be related to the model assumptions (see Agueda 2008, for more details). The maximum injection rate for Ulysses was about two orders of magnitude smaller than for $A C E$.

For the 2001/360 event, the injection in the $A C E$ flux tube started at 05:25 UT and lasted more than $3 \mathrm{hr}$. The injection onset occurred 5 minutes after the beginning of the type II radio emission and 15 minutes before the peak in SXR emission. The CME first appearance was at 05:30 UT, when the CME was at $4 R_{\odot}$. The injection profile shows average gap episodes of 4 minutes between injection amplitudes and the gap distribution extends up to 14 minutes only, with a peak at the mean gap value. On the other hand, the injection profile for Ulysses looks intermittent and patchy, starting at 05:54 UT and lasting at least until 12:00 UT. The mean gap period between injection amplitudes is 37 minutes, and the gap distribution is broad, extending up to 38 minutes and not peaking at the mean gap value. The maximum injection rate was three times smaller for Ulysses than for ACE.

The injection profiles inferred for the 2001/308 event last several hours and extend well beyond the flare impulsive phase. The association with a type II radio burst supports the scenario where electrons are provided by a CME-driven shock. If we regard the flare source as the originating place of the CME and of the associated shock, then the line through the Sun center 

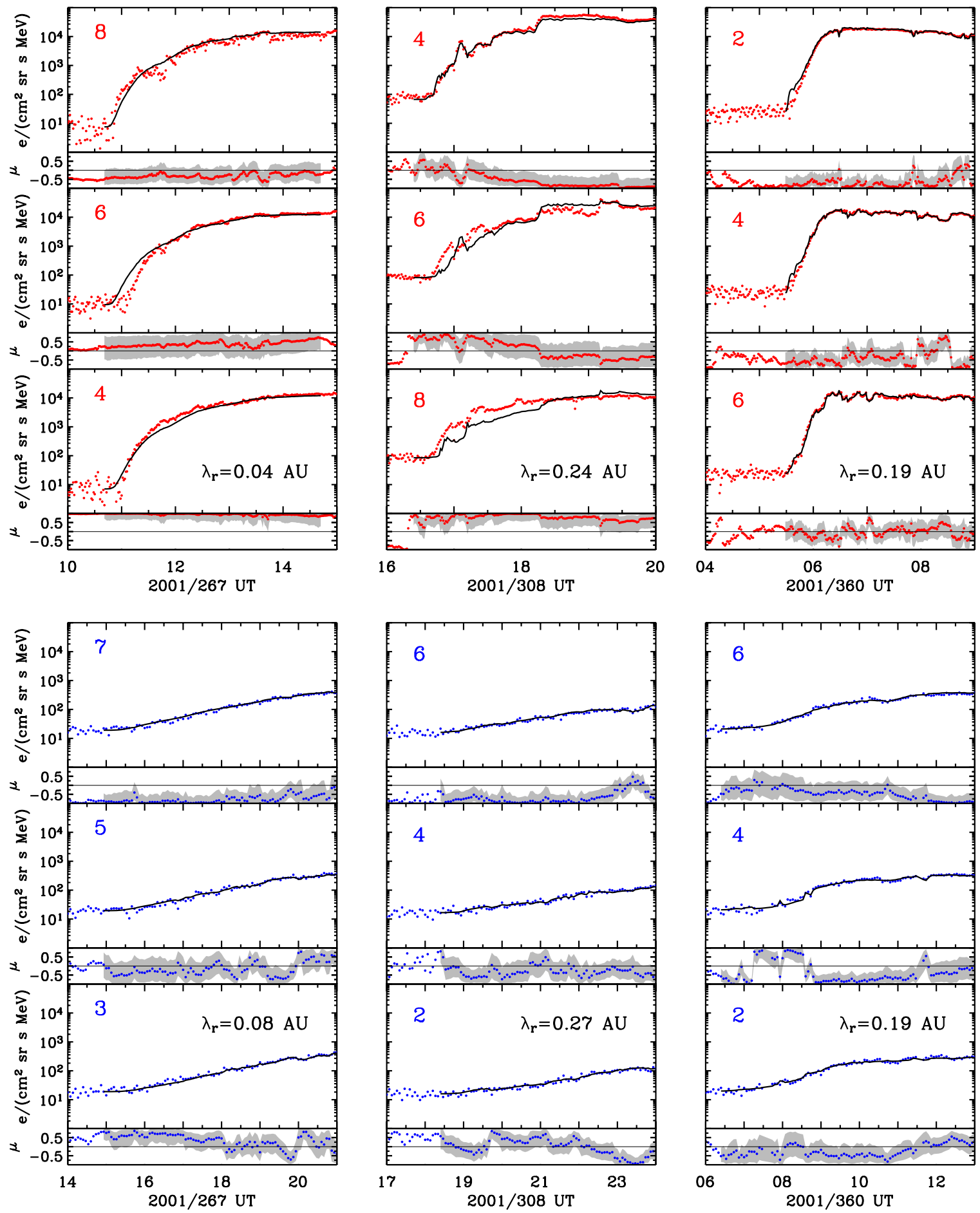

Figure 3. For each event (from top to bottom): electron sectored intensities observed by the LEFS60 telescope in three sectors in the E' 4 energy channel (the black curves show the modeled sectored intensities, the dots show the observational data: red for ACE and blue for Ulysses); pitch-angle cosine, $\mu$, scanned at the midpoint clock-angle zenith direction of the sector (colored curves) and the scanned pitch-angle cosine range (gray area).

(A color version of this figure is available in the online journal.)

and the flare source is assumed to be the shock erupting normal. Since the angular distance from the flare source to the $A C E$ footpoint was $51^{\circ}$, while the angular distance to the Ulysses footpoint was $78^{\circ}, A C E$ was most likely connected close to the nose of the shock, the fastest part of the shock front and the most efficient SEP producer (Kallenrode et al. 1993), and Ulysses was connected to the flanks. Thus, the injection profiles would reflect the fact that different relative connections to the shock front result in different injection efficiencies/histories. This scenario is supported by the earlier and larger injection rate for $A C E$ with respect to that inferred for Ulysses. Note, however, that the continuous long-duration type III radio emission observed by Wind/WAVES after 18:00 UT would not necessarily be consistent with this scenario, since it would originate from behind the CME leading edge, if one assumes the density model of Vršnak et al. (2004). For this event, the flare and 

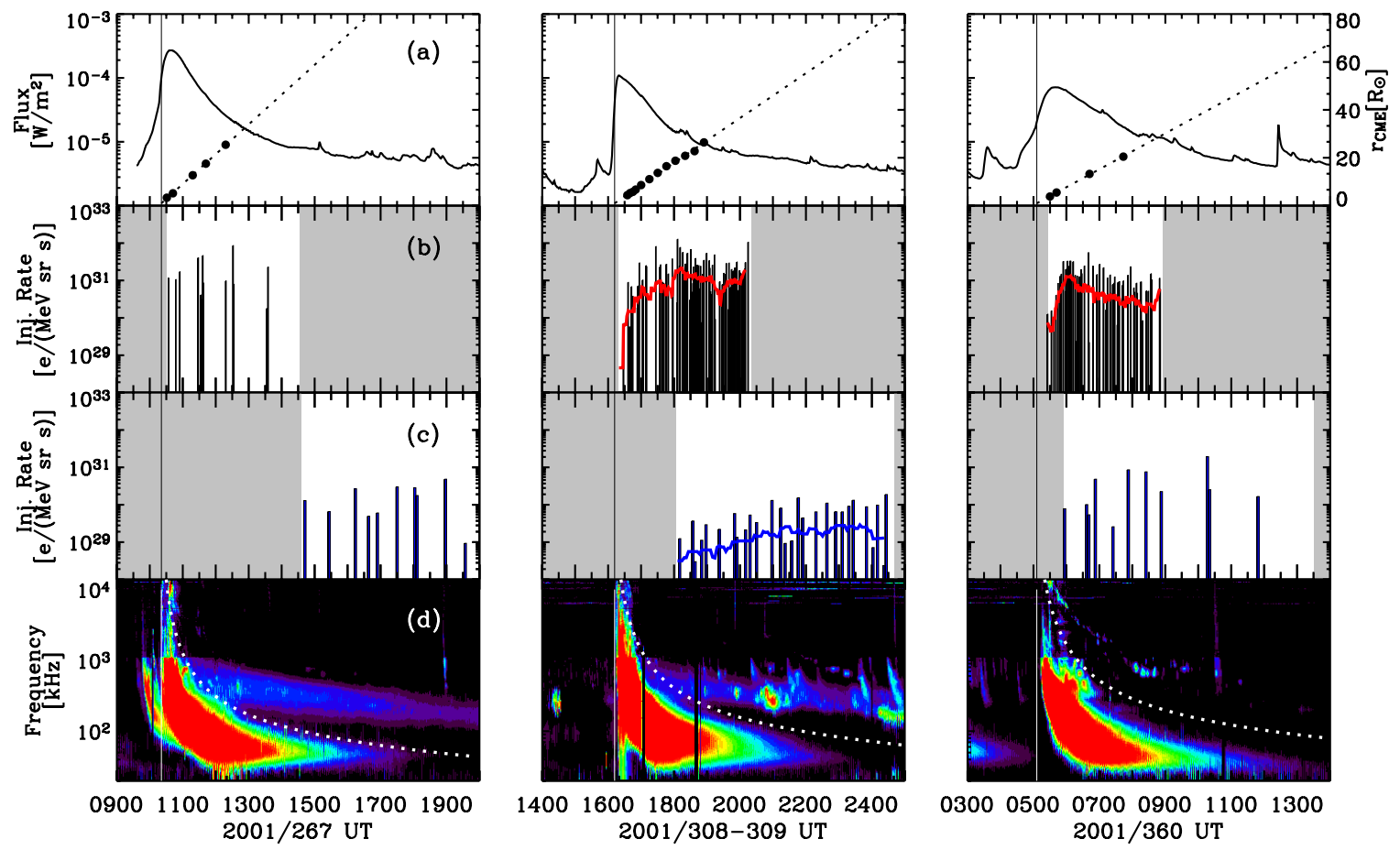

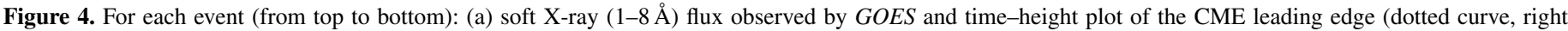

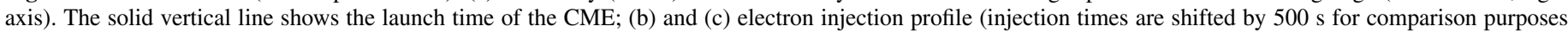

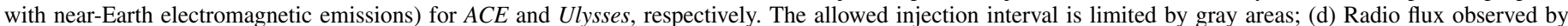
Wind/WAVES and local electron plasma frequency at the height of the CME leading edge (dotted curve).

(A color version of this figure is available in the online journal.)

the footpoints of both spacecraft were within the same magnetic field sector (see Agueda et al. 2012a, for a similar event on 2000 November 8).

The 2001/267 event provides us with an opposite showcase scenario compared with the $2001 / 308$ event, in the sense that for this event both spacecraft were connected to positions outside the flare magnetic field sector. As the 2001/308 event, the 2001/267 event was associated with a fast CME (with a speed of $2402 \mathrm{~km} \mathrm{~s}^{-1}$ ) and a strong type II radio burst. However, the injection profiles inferred for this event are not as continuous and extended as for the 2001/308 event and suggest an intermittent release of electrons well beyond the flare impulsive phase.

Finally, the injection profile inferred for the 2001/360 event shows a clear extended ( $>3 \mathrm{hr}$ ) profile for $A C E$, but shows intermittency for Ulysses. In this case, the flare and the footpoints of both spacecraft lay within the same magnetic field sector but a wrap in the HCS suggests that the CME could have been bounded by the HCS wrap. In this case, the electron release at the Ulysses footpoint could be affected by the restructuring of coronal magnetic fields due to the HCS bounding of the CME, which might have an impeding effect on the expansion of the CME-driven shock to high latitudes.

\section{DISCUSSION}

We presented a set of three large NR electron events observed by both $A C E$ and Ulysses in 2001, when Ulysses was at high latitudes and close to $2 \mathrm{AU}$. By assuming that NR electrons injected at the footpoint of the IMF line connecting the Sun with the spacecraft propagate along the magnetic field lines, we were able to reproduce the main features of the particle angular distributions and intensities observed by $A C E$ and Ulysses. The results provide an explanation of the global characteristics of the electron events, despite some minor disagreements in limited time periods and angular directions associated with fluctuations of the local interplanetary magnetic field.

For the three selected events, we found similar interplanetary transport conditions at low and at high latitudes, with values of the mean free path ranging from $0.04 \mathrm{AU}$ to $0.27 \mathrm{AU}$ (see Table 3). The events observed at 1 AU show larger anisotropies than the events observed by Ulysses near 2 AU (Lario et al. 2003). These anisotropy differences could be in part related to the different spacecraft locations, since the IMF focusing effects are much smaller at $2 \mathrm{AU}$ and the electrons reaching Ulysses had more time to isotropize as they propagated outwards in the heliosphere. In agreement with previous observational studies (e.g., Dalla et al. 2003), we found no evidence of different transport conditions in fast and slow solar wind speed regimes.

The three events were associated with large solar flares, fast CMEs and type II radio bursts. Despite the similar solar origin signatures, we found differences in the injection profiles inferred for each spacecraft. The injection timescales seemed to be ordered by the occurrence of HCS sector boundaries; that is, extended injection profiles were found if the footpoint of the spacecraft lay in the flare sector, while intermittent sparse injection episodes appeared when the spacecraft footpoint was in the opposite sector or a wrap in the HCS bounded the CME structure. Our results suggest that the large-scale coronal magnetic field might play a role in the SEP release and in the expansion of coronal shocks.

Lario \& Pick (2008) analyzed a series of difference images of the 2001/267 event as seen from the EIT telescope (Delaboudinière et al. 1995) and the C2 and C3 LASCO coronagraphs. The LASCO images showed a rapid CME propagating toward the southeast at an estimated speed of $2400 \mathrm{~km} \mathrm{~s}^{-1}$ and developing rapidly as a halo CME. The western flank of the 
CME (as also seen in EIT) propagated from the active region (located at S16E23) to about W20, where it appeared to stop. The fact that the CME/EIT structure stopped near the location of the HCS suggests that the CME could have interacted with the HCS and electron release at the distant ACE and Ulysses footpoints could have been related to reconnection processes.

Klein et al. (2005) reported several cases in which NR electron events associated with type II radio bursts showed prolonged metric-to-kilometric radio emission episodes, from several minutes to a few hours. During these time periods several emission enhancements were observed in a previously existing radio source or by a newly appearing one. This complex and prolonged radio emission is usually attributed to signatures of electron acceleration in the corona due to reconnection processes in the aftermath of CMEs during restructuring of coronal magnetic fields (e.g., Maia \& Pick 2004; Klein et al. 2005). The intermittency of the injection profiles inferred for the $2001 / 267$ event supports a scenario in which the electron release could be related to reconnection processes due to the interaction of the CME with the HCS.

Dalla et al. (2003) studied a sample of nine SEP events observed by Ulysses and near-Earth spacecraft in 2000 and 2001, which included the three events simulated in this paper. Dalla et al. (2003) performed a velocity dispersion analysis and found later SEP release times at high latitudes. The delay in particle release appeared to be correlated with the latitudinal difference between the flare and the spacecraft footpoint, rather than the total angular separation between the spacecraft footpoint and the flare. We compared the latitudinal difference between the two spacecraft footpoints for the three events under study $\left(71^{\circ}\right.$, $73^{\circ}$, and $69^{\circ}$, respectively) with the injection onset delay, but we could not infer a clear correlation because the latitudinal difference is very similar for the three events in our sample.

We do not find evidence of a faster release if the spacecraft was connected to the flare sector than if it was not, as suggested by Kallenrode (1993). The beginning of the injection at the $A C E$ footpoint starts up to 25 minutes before the SXR peak. The inferred release onset times show consistent delays at high latitudes with respect to in-ecliptic release times. The delay between the injection onset time for $A C E$ and Ulysses is of 247 minutes for the 2001/267 event, 101 minutes for the 2001/308 event, and 29 minutes for the 2001/360 event.

Details of the three-dimensional propagation of a shock in the corona remain still unknown. However three-dimensional MHD simulations provide us with evidence that the expansion of a CME-driven shock in longitude and latitude is not necessarily symmetric (Rodríguez-Gasén et al. 2011). We estimated the angular propagation speed as the ratio between the angular separation of the two spacecraft footpoints and the injection onset delay. We obtained a propagation speed of $5^{\circ} \mathrm{hr}^{-1}$ for the $2001 / 267$ event, $16^{\circ} \mathrm{hr}^{-1}$ for the 2001/308 event, and $101^{\circ} \mathrm{hr}^{-1}$ for the $2001 / 360$ event. Since the angular separation between the flare source and the $A C E$ footpoint was $80^{\circ}, 51^{\circ}$, and $12^{\circ}$ for each event, respectively, we conclude that the angular propagation speed is larger at low latitudes, which suggests a slower expansion of the release front at high latitudes (at least in the corona).

\section{CONCLUSIONS}

SEP events observed in situ by spacecraft are a result of both the injection history of particles into the spacecraft IMF flux tube and the particle transport conditions in the interplanetary medium (Cane et al. 1988; Heras et al. 1995). Magnetic connection is thus crucial when investigating the solar origin of SEP events.

In this paper, we selected a sample of three NR electron events observed by both $A C E$ and Ulysses, when it was at high latitudes and close to $2 \mathrm{AU}$. All three events were associated with large solar flares and accompanied by wide $\left(>212^{\circ}\right)$ and fast $\left(>1400 \mathrm{~km} \mathrm{~s}^{-1}\right.$ ) CMEs. Strong decametric type II radio bursts, often considered as a clear signature of a propagating shock, were reported during the three events. The different relative positions between the flare site and the footpoints of the two spacecraft with respect to the HCS allowed us to study the role of the HCS in NR electron release for these three events.

We found indications that the HCS could be playing a role in NR electron release and regulate the characteristics of the electron injection profiles. Extended injection profiles, probably associated with coronal shocks, are found if the magnetic footpoints of the spacecraft lay in the same magnetic sector as the associated flare. On the other hand, intermittent sparse injection episodes appear when the spacecraft footpoints are in the opposite sector or a wrap in the HCS bounded the CME structure, which suggests that in these cases reconnection processes could be playing a role in releasing NR electrons into the spacecraft flux tubes.

An extension of this sample, including other multi-spacecraft events, would be necessary to gain definitive understanding of the processes involved in SEP release. Moreover the analysis of a larger number of events would contribute to obtaining a more detailed description of the two classes of injection referred in this paper as "sparse" and "extended." SEPServer, ${ }^{10}$ a three year collaborative project funded by the Seventh Framework Programme (FP7-SPACE) of the European Union, aims at building an Internet server with in situ measurements of solar energetic particles and associated electromagnetic emissions. SEPServer will also provide registered users with a large database of electron Green's functions (Agueda et al. 2012b) and the inversion software for particle observations by $A C E$, Ulysses, Wind, and STEREO, which will greatly facilitate a broadening of the sample of modeled multi-spacecraft events (e.g., Malandraki et al. 2012). We believe that uncertainties in SEP release and interplanetary transport will be further reduced with SEP observations closer to the Sun by Solar Orbiter and Solar Probe Plus.

The research leading to these results has received funding from the European Union's Seventh Framework Programme (FP7/2007-2013) under grant agreement No. 262773 (SEPServer). Computational support was provided by the Centre de Serveis Científics i Acadèmics de Catalunya (CESCA). N.A. and B.S. were partially supported by the Ministerio de Economía i Competitividad (Spain), under the project AYA2010-17286. N.A. and B.S. acknowledge support by the COST Action ES0803 "Developing space weather products and services in Europe." D.L. acknowledges the support from NASA under grants NNX09AG30G and NNX11A083G.

\section{REFERENCES}

Agueda, N. 2008, PhD thesis, Univ. Barcelona, http://hdl.handle.net/10803/749 Agueda, N., Lario, D., Ontiveros, V., et al. 2012a, SoPh, 281, 319

Agueda, N., Lario, D., Vainio, R., et al. 2009a, A\&A, 507, 981

Agueda, N., Vainio, R., Lario, D., \& Sanahuja, B. 2008, ApJ, 675, 1601

Agueda, N., Vainio, R., Lario, D., \& Sanahuja, B. 2009b, AdSpR, 44, 794

\footnotetext{
10 http://wwww.sepserver.eu/
} 
Agueda, N., Vainio, R., \& Sanahuja, B. 2012b, ApJS, 202, 18

Bougeret, J.-L., Kaiser, M. L., Kellogg, P. J., et al. 1995, SSRv, 71, 231

Cane, H. V., \& Erickson, W. C. 2005, ApJ, 623, 1180

Cane, H. V., Reames, D. V., \& von Rosenvinge, T. T. 1988, JGR, 93, 9555

Cane, H. V., Stone, R. G., Fainberg, J., et al. 1981, GeoRL, 8, 1285

Dalla, S., Balogh, A., Krucker, S., et al. 2003, AnGeo, 21, 1367

Delaboudinière, J.-P., Artzner, G. E., Brunaud, J., et al. 1995, SoPh, 162, 291

Dröge, W. 2003, ApJ, 589, 1027

Dröge, W., Kartavykh, Y. Y., Klecker, B., \& Kovaltsov, G. A. 2010, ApJ, 709,912

Gold, R. E., Krimigis, S. M., Hawkins, S. E., III, et al. 1998, SSRv, 86, 541

Gopalswamy, N., Yashiro, S., Michałek, G., et al. 2002, ApJL, 572, L103

Heras, A. M., Sanahuja, B., Lario, D., et al. 1995, ApJ, 445, 497

Hoeksema, J. T., Wilcox, J. M., \& Scherrer, P. H. 1983, JGR, 88, 9910

Jokipii, J. R. 1966, ApJ, 146, 480

Kahler, S. W. 2007, SSRv, 129, 359

Kahler, S. W., Kunches, J. M., \& Smith, D. F. 1996, JGR, 101, 24383

Kallenrode, M.-B. 1993, JGR, 98, 19037

Kallenrode, M.-B., Wibberenz, G., \& Hucke, S. 1992, ApJ, 394, 351

Kallenrode, M.-B., Wibberenz, G., Kunow, H., et al. 1993, SoPh, 147, 377

Kelly, J., Dalla, S., \& Laitinen, T. 2012, ApJ, 750, 47
Klein, K.-L., Krucker, S., Trottet, G., \& Hoang, S. 2005, A\&A, 431, 1047

Lanzerotti, L. J., Gold, R. E., Anderson, K. A., et al. 1992, A\&AS, 92, 349

Lario, D., \& Pick, M. 2008, in The Heliosphere through the Solar Activity Cycle, ed. A. Balogh, L. J. Lanzerotti, \& S. T. Suess (Berlin: Springer), 151

Lario, D., Roelof, E. C., Decker, R. B., \& Reisenfeld, D. B. 2003, AdSpR, 32 , 579

Lawson, C. L., \& Hanson, R. J. 1974, Solving Least Squares Problems (Englewood Cliffs: Prentice-Hall)

Maia, D. J. F., \& Pick, M. 2004, ApJ, 609, 1082

Malandraki, O. E., Agueda, N., Papaioannou, A., et al. 2012, SoPh, 281, 333

McComas, D. J., Elliott, H. A., Gosling, J. T., et al. 2002, GeoRL, 29, 1290

Mullan, D. J. 1981, Proc. 17th Int. Cosmic Ray Conf. (Paris), 3, 51

Rodríguez-Gasén, R., Aran, A., Sanahuja, B., Jacobs, C., \& Poedts, S. 2011, AdSpR, 47, 2140

Roelof, E. C. 1969, in Lectures in High-Energy Astrophysics, ed. H. Ögelman \& J. R. Wayland (NASA SP-199; Washington, DC: NASA), 111

Ruffolo, D. 1995, ApJ, 442, 861

Schatten, K. H., Wilcox, J. M., \& Ness, N. F. 1969, SoPh, 6, 442

Steinolfson, R. S., \& Mullan, D. J. 1980, ApJ, 241, 1186

Vršnak, B., Magdalenić, J., \& Zlobec, P. 2004, A\&A, 413, 753

Zhao, X., Feng, X., \& Wu, C.-C. 2007, JGR, 112, 6107 